Research Paper

\title{
Serum Endocan as a Novel Prognostic Biomarker in Patients with Hepatocellular Carcinoma
}

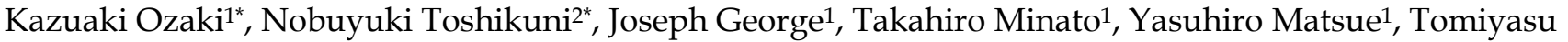 \\ Arisawa ${ }^{2}$ and Mikihiro Tsutsumi ${ }^{\bowtie}$ \\ 1. Department of Hepatology, Kanazawa Medical University, Uchinada, Ishikawa, Japan; \\ 2. Department of Gastroenterology, Kanazawa Medical University, Uchinada, Ishikawa, Japan. \\ * Contributed equally. \\ $\triangle$ Corresponding author: Dr. Nobuyuki Toshikuni, Department of Gastroenterology, Kanazawa Medical University, 1-1 Daigaku, \\ Uchinada-machi, Kahoku, Ishikawa 920-0293, Japan. E-mail: n.toshikuni@gmail.com. \\ (c) Ivyspring International Publisher. This is an open-access article distributed under the terms of the Creative Commons License (http://creativecommons.org/ \\ licenses/by-nc-nd/3.0/). Reproduction is permitted for personal, noncommercial use, provided that the article is in whole, unmodified, and properly cited.
}

Received: 2013.09.17; Accepted: 2014.02.09; Published: 2014.03.04

\begin{abstract}
Endocan is a vascular endothelium-derived factor regulated by angiogenic factors. The aim of this study was to determine whether serum endocan levels are prognostic for survival in patients with hepatocellular carcinoma $(\mathrm{HCC})$. Serum endocan levels were measured in $64 \mathrm{HCC}$ patients who were naïve to treatment, eight apparently healthy subjects, and 68 patients with liver cirrhosis; the latter two groups served as controls. Prognostic factors for the survival of HCC patients were examined using a Cox proportional hazards model. The median serum endocan levels were $\mathrm{I} .145$ $\mathrm{ng} / \mathrm{mL}$ (range, $0.93-1.68 \mathrm{ng} / \mathrm{mL}$ ) in healthy subjects, $1.93 \mathrm{ng} / \mathrm{mL}$ (range, $0.45-8.47 \mathrm{ng} / \mathrm{mL}$ ) in liver cirrhosis patients, and $3.73 \mathrm{ng} / \mathrm{mL}$ (range, $0.74-10.95 \mathrm{ng} / \mathrm{mL}$ ) in HCC patients $(P=0.000 \mathrm{I})$. In HCC patients, elevated serum endocan levels were significantly associated with poor hepatic function $(P$ $=0.015)$, a greater number of tumors $(P=0.034)$, and vascular invasion $(P=0.043)$. The median follow-up period was 23.0 months, and $33 \mathrm{HCC}$ patients died during follow up. Multivariate analysis showed that serum endocan levels $\geq 2.20 \mathrm{ng} / \mathrm{mL}$ (hazard ratio $2.36,95 \%$ confidence interval 1.22-5.36, $P=0.008$ ) as well as elevated serum $\alpha$-fetoprotein and des-y-carboxy prothrombin levels were independent prognostic biomarkers for poor survival. The combination of serum endocan and these two additional markers was significantly predictive of worse survival $(P<$ $0.000 \mathrm{I}$ ). Thus, serum endocan may be a prognostic biomarker for survival in HCC patients, and the combination of serum endocan, $\alpha$-fetoprotein, and des- $\gamma$-carboxy prothrombin levels can result in better prognostic stratification of these patients.
\end{abstract}

Key words: hepatocellular carcinoma, endocan, prognostic survival.

\section{Introduction}

Hepatocellular carcinoma (HCC) is a global health problem because of its increasing prevalence worldwide and its poor prognosis. In fact, HCC ranks as the third leading cause of cancer-related mortality (1). Various therapeutic measures, including surgical resection, transplantation, radiofrequency ablation (RFA), transarterial chemoembolization (TACE), hepatic arterial infusion chemotherapy, and molecular targeted therapy, are used to treat patients with HCC (2). Although treatment selection is generally based on cancer stage and liver function, administration of the same treatment in HCC patients with similar conditions can result in a wide range of outcomes. Thus, prognostic and predictive biomarkers are needed for the optimal management of patients with HCC.

HCCs are typically hypervascular tumors, and HCC-derived angiogenic factors, such as vascular endothelial growth factor (VEGF) and fibroblast growth factor, are closely associated with tumor pro- 
gression. The expression levels of these factors in tumors and sera have been shown to be predictive of recurrence and survival $(3,4)$. In contrast, less is known about the vascular endothelium-derived factors regulated by these HCC-derived angiogenic factors.

Endocan, or endothelial specific molecule-1, is a soluble proteoglycan of $50 \mathrm{kDa}$ that is synthesized and secreted by activated vascular endothelial cells, including tumor endothelial cells (5-8). Endocan production is upregulated by angiogenic factors and inflammatory cytokines, such as tumor necrosis factor- $\alpha$ and interleukin-1 $\beta(5,9)$. In experimental studies, endocan has been found to induce tumor formation and to be closely associated with the conversion of dormant tumors into fast-growing angiogenic tumors $(10,11)$. Recent studies have shown that endocan is overexpressed at the mRNA and/or protein levels in various tumor types, including glioblastoma, pituitary adenoma, non-small cell lung cancer, gastric cancer, colorectal cancer, renal cell cancer, bladder cancer, ovarian cancer, and HCC $(9,12-23)$. Moreover, endocan overexpression in cancer tissues and sera has been associated with tumor progression and poor outcomes (13-17, 19-23). A study of the association between endocan expression and HCC patient outcomes found that microvessel density in surgically resected tumors, represented by endocan expression, was prognostic for worse survival (21). More recent studies on HCC showed that elevated serum endocan levels and endocan expression by stromal endothelial cells in HCC tissues were predictive of recurrence after RFA $(24,25)$.

The aim of the present study was to determine whether serum endocan is a prognostic biomarker for survival and tumor recurrence in HCC patients. Our findings suggested that serum endocan may serve as a prognostic biomarker for survival in HCC patients and that the combination of serum endocan with the serum tumor markers $\alpha$-fetoprotein (AFP) and des- $\gamma$-carboxy prothrombin (DCP) can result in improved prognostic stratification of these patients.

\section{Materials and Methods}

\section{Patients}

This study enrolled 64 treatment-naïve HCC patients with liver cirrhosis (LC), including 41 men and 23 women, with a median age of 71 years (range, 47-86 years). The patients were admitted between June 1995 and March 2012. The control group included eight apparently healthy subjects, including five men and three women with a median age of 71 years (range, 46-81 years), and 68 patients with LC, including 36 men and 32 women with a median age of
64 years (range, 27-85 years). Each patient and/or a member of his/her family provided written informed consent. The study protocol was approved by the Ethics Committee of Kanazawa Medical University (approval no. 217) and was conducted in accordance with the Declaration of Helsinki.

Serum specimens were obtained before treatment and were stored at $-80^{\circ} \mathrm{C}$ until analysis. LC was diagnosed based on laboratory data and imaging findings. HCC was diagnosed based on imaging findings and serum levels of AFP and DCP or by histological examination of biopsied specimens. HCCs were staged according to the tumor-node-metastasis (TNM) classification proposed by the Liver Cancer Study Group of Japan (26). Briefly, T1 tumors were defined as those meeting all three of the following requirements: [1] solitary tumors; [2] size $\leq 2 \mathrm{~cm}$; and [3] no vascular invasion of the portal vein, hepatic vein, or bile duct. T2, T3, and T4 tumors met 2,1 , and 0 requirements, respectively. Stage I, II, III, IVA, and IVB tumors were defined as T1 N0 M0; T2 N0 M0; T3 N0 M0; T4 N0 M0 or any T N1 M0; and any T/N M1, respectively.

\section{HCC treatment and follow-up}

Treatment was consistent in principle with the Evidence-Based Clinical Practice Guidelines for Hepatocellular Carcinoma in Japan (the J-HCC Guidelines) (27). RFA was performed with a cool-tip RFA system (Covidien, Mansfield, MA, USA). TACE was performed by injecting an emulsion of epirubicin hydrochloride in iodized oil, zinostatin stimalamer, or a miriplatin suspension in iodized oil into the feeding arteries of the tumor, followed by gelatin sponges. Hepatic arterial infusion chemotherapy was performed using low-dose 5-fluorouracil and cisplatin. Sorafenib was administered as a molecular targeted therapy. Surgical resection and RFA were defined as curative treatments; all other treatments were defined as non-curative. After treatment, the patients underwent laboratory tests, including measurement of serum tumor markers every 1-3 months and imaging examinations every 3-6 months. When recurrent HCCs were detected, the appropriate treatment options were chosen based on the treatment policy.

\section{Measurement of serum endocan, VEGF, and tumor marker levels}

Serum endocan levels were measured using an endocan enzyme-linked immunosorbent assay (ELISA) kit (EndoMark H1; Lunginnov s.a.s., Lille, France) according to the manufacturer's instructions. Briefly, each microwell plate was coated with $100 \mu \mathrm{L}$ of a capture antibody $(2 \mu \mathrm{g} / \mathrm{mL})$, and the plates were incubated overnight at $4^{\circ} \mathrm{C}$. The plates were then 
blocked with $0.1 \%$ bovine serum albumin in phosphate-buffered saline for $1 \mathrm{~h}$ at room temperature. After washing, $100 \mu \mathrm{L}$ of the serum specimens (1:4 dilution) or standards were added to each well, followed by incubation overnight at $4^{\circ} \mathrm{C}$. Biotinylated secondary antibody $(100 \mu L ; 1: 10,000)$ was then dispensed into each well and incubated for $1 \mathrm{~h}$ at room temperature. After washing, $100 \mu \mathrm{L}$ of streptavidin-horseradish peroxidase $(1: 10,000)$ was added, and the bound endocan was detected by the chromogenic reaction of tetramethylbenzidine substrate solution in the presence of horseradish peroxidase. The optical density was determined using a microplate reader set to $450 \mathrm{~nm}$ and with a wavelength correction set to 630 $\mathrm{nm}$. Each serum sample was assayed for endocan in triplicate, and the median value was used as the result. To examine the relationships between endocan and angiogenic factors, serum VEGF levels were measured using a VEGF ELISA kit (Life Technologies, Carlsbad, CA, USA) according to the manufacturer's protocol. Serum AFP and DCP levels were measured by chemiluminescent enzyme immunoassays using commercially available kits.

\section{Statistical analysis}

Baseline data from the patients are expressed as medians (ranges). The Mann-Whitney $U$ test or the Kruskal-Wallis test was used to compare continuous variables, and Fisher's exact probability test was used to compare categorical variables. Spearman's rank correlation coefficient was used to assess the associations between serum endocan levels, patient age, and serum VEGF, AFP, and DCP levels. Because platelets have been reported to be the main transporters of circulating VEGF $(28,29)$, serum VEGF levels were corrected by dividing them by platelet counts (VEGF/platelets). Receiver operating characteristic curve analysis was used to distinguish between HCC and LC, with the optimal cut-off value based on the Youden index. Prognostic factors in HCC patients were evaluated using the Cox proportional hazard model, with the following variables entered into the model: age (<70 vs. $\geq 70$ years), gender (female vs. male), etiology of liver disease (non-viral vs. viral), tumor stage (stage I/II vs. III/IV), Child-Pugh grade (A vs. B/C), treatment option (curative vs. non-curative), and serum levels of endocan $(<2.20 \mathrm{vs}$. $\geq 2.20 \mathrm{ng} / \mathrm{mL}$ ), VEGF/platelets $(<2.18$ vs. $\geq 2.18$ $\left.\mathrm{pg} / 10^{6}\right), \operatorname{AFP}(<100 \mathrm{vs} . \geq 100 \mathrm{ng} / \mathrm{mL})$, and DCP $(<26$ vs. $\geq 26 \mathrm{mAU} / \mathrm{mL})$. To determine the cut-off values for serum endocan, DCP, and VEGF/platelets, we tested whether the $25 \mathrm{th}, 50 \mathrm{th}$, or 75 th percentile values maximized the hazard ratios. Consequently, the 25th percentiles were chosen as the cut-off values for both serum endocan and DCP, whereas the 75th per- centile was chosen as the cut-off value for VEGF/platelets. A cut-off value of $100 \mathrm{ng} / \mathrm{mL}$ was used for serum AFP on the basis of clinical data from previous studies (30). The model also included the number of elevated serum markers as a variable. Univariate analysis was performed for each variable, followed by multivariate analysis. The overall survival rates were calculated using the Kaplan-Meier method, and differences were evaluated using the log-rank test. $P<0.05$ was considered statistically significant. All statistical analyses were performed using Stata version 11.1 (StataCorp, College Station, TX, USA).

\section{Results}

\section{Serum endocan levels in non-HCC and HCC patients}

The median serum endocan levels were 1.145 $\mathrm{ng} / \mathrm{mL}$ (range, 0.93-1.68 $\mathrm{ng} / \mathrm{mL}$ ) in apparently healthy subjects, $1.93 \mathrm{ng} / \mathrm{mL}$ (range, 0.45-8.47 $\mathrm{ng} / \mathrm{mL}$ ) in LC patients, and $3.73 \mathrm{ng} / \mathrm{mL}$ (range, $0.74-10.95 \mathrm{ng} / \mathrm{mL}$ ) in HCC patients (Fig. 1). Serum endocan levels were not associated with age, gender, or etiology of liver disease but were positively associated with the Child-Pugh grade in both LC and HCC patients (Fig. 2). Serum endocan levels were higher in HCC versus LC patients at each Child-Pugh grade: $3.38 \mathrm{ng} / \mathrm{mL}$ (range, $0.74-10.95 \mathrm{ng} / \mathrm{mL}$ ) vs. 1.63 $\mathrm{ng} / \mathrm{mL}$ (range, $0.45-4.35 \mathrm{ng} / \mathrm{mL}$ ), respectively, for Child-Pugh A; $5.61 \mathrm{ng} / \mathrm{mL}$ (range, $0.74-9.44 \mathrm{ng} / \mathrm{mL}$ ) vs. $2.59 \mathrm{ng} / \mathrm{mL}$ (range, $0.65-6.88 \mathrm{ng} / \mathrm{mL}$ ), respective$\mathrm{ly}$, for Child-Pugh $\mathrm{B}$; and $8.475 \mathrm{ng} / \mathrm{mL}$ (range. $3.53-9.33 \mathrm{ng} / \mathrm{mL}$ ) vs. $3.08 \mathrm{ng} / \mathrm{mL}$ (range, $1.54-8.47$ $\mathrm{ng} / \mathrm{mL}$ ), respectively, for Child-Pugh C. The distribution of Child-Pugh grade did not differ significantly between LC and HCC patients. Receiver operating characteristic curve analysis showed that a serum endocan level cut-off value of $3.59 \mathrm{ng} / \mathrm{mL}$ discriminated between HCC and LC with $54.7 \%$ sensitivity and $86.8 \%$ specificity (Fig. 3 ).

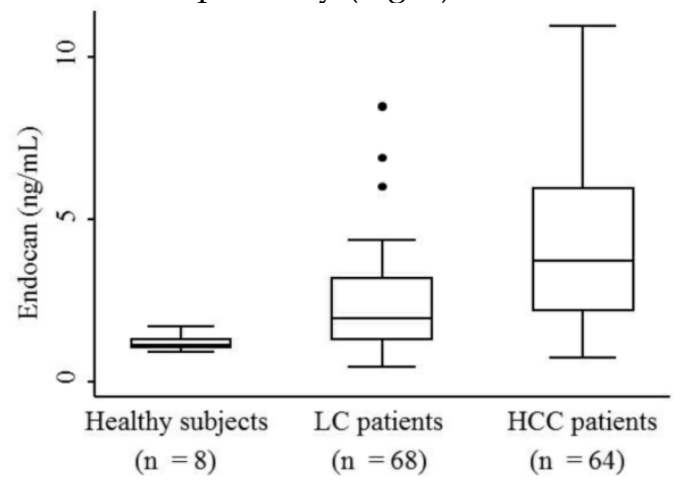

Figure I. Serum endocan levels in HCC patients and control groups. Endocan levels were highest in $\mathrm{HCC}$ patients, somewhat lower in LC patients, and lowest in apparently healthy subjects $(P=0.000 \mathrm{I})$. $\mathrm{HCC}$, hepatocellular carcinoma; $\mathrm{LC}$, liver cirrhosis. 


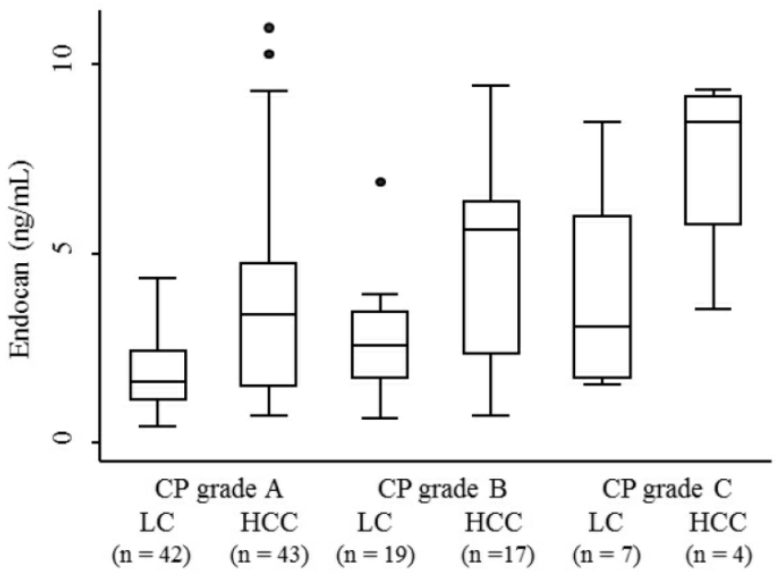

Figure 2. Serum endocan levels in LC and HCC patients at each CP grade. Serum endocan levels were positively associated with CP grade in both LC $(P=$ $0.018)$ and HCC $(P=0.024)$ patients. At each $C P$ grade, endocan levels were higher in $\mathrm{HCC}$ than in $\mathrm{LC}$ patients (grade $\mathrm{A}, P=0.0002$; grade $B, P=0.015$; grade $C, P=0.059)$. $L C$, liver cirrhosis; $H C C$, hepatocellular carcinoma; $C P$, Child-Pugh.

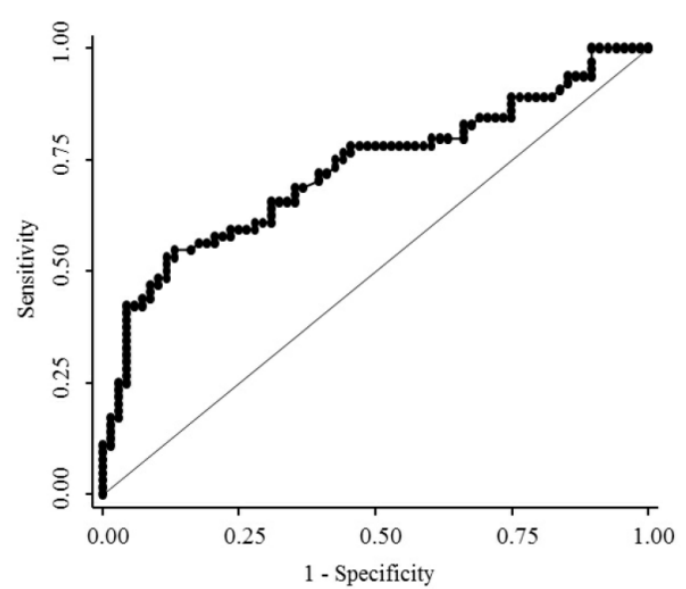

Figure 3. Receiver operating characteristic curve analysis for discrimination between HCC and LC. The area under the curve was 0.726 ( $95 \%$ confidence interval $0.643-0.80 \mathrm{I})$. HCC, hepatocellular carcinoma; LC, liver cirrhosis.

\section{Association of serum endocan levels with clinical variables and tumor markers}

Table 1 lists the baseline characteristics of the 64 HCC patients. When these patients were divided into two groups based on a cut-off serum endocan level of $2.20 \mathrm{ng} / \mathrm{mL}$, the prevalence of Child-Pugh grade B/C, number of tumors $(\geq 4)$, and vascular invasion of HCC tended to be higher in patients with higher versus lower endocan levels. The prevalence of non-curative treatment and serum AFP levels were significantly higher in patients with higher endocan levels; however, VEGF/platelets and serum DCP levels did not differ significantly between the two groups. In contrast, the prevalence of higher numbers of tumors or vascular invasion did not differ between patients with Child-Pugh grade A and those with grades B/C. When these patients were divided into two groups according to clinical variables, serum endocan levels were significantly higher in patients with Child-Pugh grade $B / C$ versus Child-Pugh grade $A$, in patients with number of tumors $(\geq 4)$ versus number of tumors (1-3), and in patients with versus without vascular invasion of HCC (Table 2). Serum endocan levels were correlated significantly with serum AFP $(\rho=$ $0.334, P=0.007$, Spearman's rank correlation coefficient) levels but not with VEGF/platelets $(\rho=0.046, P$ $=0.720)$ or serum DCP $(\rho=-0.029, P=0.818)$ levels.

Table I. Baseline characteristics of HCC patients $(n=64)$.

\begin{tabular}{|c|c|c|c|c|}
\hline \multirow[t]{2}{*}{ Variable } & \multirow{2}{*}{$\begin{array}{l}\text { Total cohort } \\
(\mathrm{n}=64)\end{array}$} & \multirow{2}{*}{$\begin{array}{l}\text { Serum endocan }<2.20 \mathrm{ng} / \mathrm{mL} \\
(\mathrm{n}=16)\end{array}$} & \multirow{2}{*}{$\begin{array}{l}\text { Serum endocan } \geq 2.20 \mathrm{ng} / \mathrm{mL} \\
(\mathrm{n}=48)\end{array}$} & \multirow[t]{2}{*}{$P$-value } \\
\hline & & & & \\
\hline Age (years) & $71(47-86)$ & $70.5(51-85)$ & $71(47-86)$ & 0.798 \\
\hline$<70 / \geq 70$ & $29 / 35$ & $8 / 8$ & $21 / 27$ & 0.774 \\
\hline Gender, Male/Female & $41 / 23$ & $11 / 5$ & $30 / 18$ & 0.768 \\
\hline \multicolumn{5}{|l|}{ Etiology of liver disease } \\
\hline HBV/HCV/Non-viral & $10 / 40 / 14$ & $1 / 11 / 4$ & $9 / 29 / 10$ & \\
\hline Viral/Non-viral & $50 / 14$ & $12 / 4$ & $38 / 10$ & 0.736 \\
\hline \multicolumn{5}{|l|}{ Child-Pugh grade } \\
\hline $\mathrm{A} / \mathrm{B} / \mathrm{C}$ & $43 / 17 / 4$ & $14 / 2 / 0$ & $29 / 15 / 4$ & \\
\hline $\mathrm{A} / \mathrm{B}$ or $\mathrm{C}$ & $43 / 21$ & $14 / 2$ & $29 / 19$ & 0.065 \\
\hline Maximal tumor size $(\mathrm{cm})$ & $2.9(1.0-16.0)$ & $2.7(1.0-5.9)$ & $3.05(1.0-16.0)$ & 0.268 \\
\hline$<3 / \geq 3$ & $33 / 31$ & $10 / 6$ & $23 / 25$ & 0.392 \\
\hline \multicolumn{5}{|l|}{ Number of tumors } \\
\hline $1 / 2 / 3 / \geq 4$ & $42 / 10 / 2 / 10$ & $11 / 4 / 1 / 0$ & $31 / 6 / 1 / 10$ & \\
\hline $1-3 / \geq 4$ & $54 / 10$ & $16 / 0$ & $38 / 10$ & 0.055 \\
\hline \multicolumn{5}{|l|}{ Vascularity of HCC } \\
\hline Hypovascular/Hypervascular & $6 / 58$ & $3 / 13$ & $3 / 45$ & 0.160 \\
\hline Vascular invasion of HCC, No/Yes & $53 / 11$ & $16 / 0$ & $37 / 11$ & 0.052 \\
\hline
\end{tabular}




\begin{tabular}{|c|c|c|c|c|}
\hline \multirow[t]{2}{*}{ Variable } & \multirow{2}{*}{$\frac{\text { Total cohort }}{(\mathrm{n}=64)}$} & \multirow{2}{*}{$\begin{array}{l}\text { Serum endocan }<2.20 \mathrm{ng} / \mathrm{mL} \\
(\mathrm{n}=16)\end{array}$} & \multirow{2}{*}{$\begin{array}{l}\text { Serum endocan } \geq 2.20 \mathrm{ng} / \mathrm{mL} \\
(\mathrm{n}=48)\end{array}$} & \multirow[t]{2}{*}{$P$-value* } \\
\hline & & & & \\
\hline Portal vein invasion, No/Yes & $53 / 11$ & $16 / 0$ & $37 / 11$ & \\
\hline Hepatic vein invasion, No/Yes & $60 / 4$ & $16 / 0$ & $44 / 4$ & \\
\hline Extrahepatic spread, No/Yes & $59 / 5$ & $16 / 0$ & $43 / 5$ & 0.320 \\
\hline \multicolumn{5}{|l|}{ Tumor stage } \\
\hline I/II/III/IV & $18 / 23 / 11 / 12$ & $5 / 6 / 5 / 0$ & $13 / 17 / 6 / 12$ & \\
\hline I or II/III or IV & $41 / 23$ & $11 / 5$ & $30 / 18$ & 0.768 \\
\hline \multicolumn{5}{|l|}{ Treatment option } \\
\hline Curative & 38 & 14 & 24 & \\
\hline Surgical resection/RFA & $8 / 30$ & $5 / 9$ & $3 / 21$ & \\
\hline Non-curative & 26 & 2 & 24 & \\
\hline TACE/HAIC/Sorafenib & $16 / 5 / 5$ & $2 / 0 / 0$ & $14 / 5 / 5$ & \\
\hline Curative/Non-curative & $38 / 26$ & $14 / 2$ & $24 / 24$ & 0.009 \\
\hline Serum endocan $(\mathrm{ng} / \mathrm{mL})$ & $3.73(0.74-10.95)$ & $1.275(0.74-2.17)$ & $4.715(2.2-10.95)$ & $<0.0001$ \\
\hline$<2.20 / \geq 2.20$ & $16 / 48$ & $16 / 0$ & $0 / 48$ & \\
\hline VEGF/platelets (pg/106) & $1.19(0.35-9.74)$ & $0.98(0.35-6.28)$ & $1.33(0.36-9.74)$ & 0.299 \\
\hline$<2.18 / \geq 2.18$ & $48 / 16$ & $13 / 3$ & $35 / 13$ & 0.741 \\
\hline Serum AFP (ng/mL) & $19.0(1.6-19353)$ & $7.5(2.4-2630)$ & $25.0(1.6-19353)$ & 0.021 \\
\hline$<100 / \geq 100$ & $44 / 20$ & $13 / 3$ & $31 / 17$ & 0.351 \\
\hline Serum DCP (mAU/mL) & $119(14-121000)$ & $264(17-10700)$ & $119(14-121000)$ & 0.969 \\
\hline$<26 / \geq 26$ & $16 / 48$ & $3 / 13$ & $13 / 35$ & 0.741 \\
\hline
\end{tabular}

HCC, hepatocellular carcinoma; HBV, hepatitis B virus; HCV, hepatitis C virus; RFA, radiofrequency ablation; TACE, transarterial chemoembolization; HAIC, hepatic arterial infusion chemotherapy; VEGF, vascular endothelial growth factor; AFP, $\alpha$-fetoprotein; DCP, des- $\gamma$-carboxy prothrombin.

* Mann-Whitney $U$-test for continuous variables, Fisher's exact probability test for categorical variables.

Table 2. Association of serum endocan levels with clinical variables in HCC patients $(n=64)$.

\begin{tabular}{|c|c|c|c|c|}
\hline Variable & Category & $\begin{array}{l}\text { Number of } \\
\text { patients }\end{array}$ & $\begin{array}{l}\text { Median } \\
\text { serum } \\
\text { endocan } \\
(\mathrm{ng} / \mathrm{mL})\end{array}$ & $P$-value* \\
\hline \multirow[t]{2}{*}{ Age (years) } & $<70$ & 29 & 3.97 & 0.642 \\
\hline & $\geq 70$ & 35 & 3.59 & \\
\hline \multirow[t]{2}{*}{ Gender } & Female & 23 & 3.63 & 0.928 \\
\hline & Male & 41 & 3.79 & \\
\hline \multirow{2}{*}{$\begin{array}{l}\text { Etiology of liver } \\
\text { disease }\end{array}$} & Non-viral & 14 & 4.05 & 0.620 \\
\hline & Viral & 50 & 3.65 & \\
\hline \multirow[t]{2}{*}{ Child-Pugh grade } & A & 43 & 3.38 & 0.015 \\
\hline & $\mathrm{B} / \mathrm{C}$ & 21 & 5.48 & \\
\hline \multirow{2}{*}{$\begin{array}{l}\text { Maximal tumor size } \\
(\mathrm{cm})\end{array}$} & $<3$ & 33 & 4.54 & 0.809 \\
\hline & $\geq 3$ & 31 & 3.59 & \\
\hline \multirow[t]{2}{*}{ Number of tumors } & $1-3$ & 54 & 3.46 & 0.034 \\
\hline & $\geq 4$ & 10 & 4.94 & \\
\hline \multirow[t]{2}{*}{ Vascularity of HCC } & Hypovascular & 6 & 2.05 & 0.144 \\
\hline & Hypervascular & 58 & 3.88 & \\
\hline \multirow{2}{*}{$\begin{array}{l}\text { Vascular invasion of } \\
\text { HCC }\end{array}$} & Absent & 53 & 3.38 & 0.043 \\
\hline & Present & 11 & 4.76 & \\
\hline \multirow[t]{2}{*}{ Extrahepatic spread } & Absent & 59 & 3.63 & 0.074 \\
\hline & Present & 5 & 6.74 & \\
\hline \multirow[t]{2}{*}{ Tumor stage } & $\mathrm{I} / \mathrm{II}$ & 41 & 3.38 & 0.240 \\
\hline & III/IV & 23 & 3.97 & \\
\hline
\end{tabular}

HCC, hepatocellular carcinoma.

* Mann-Whitney U-test.

\section{Serum endocan levels as a prognostic biomarker of survival}

The median follow-up period for the 64 HCC patients was 23.0 months (range, 2.0-109 months). A total of nine patients were lost to follow-up, with 5,1 ,
1 , and 2 patients lost at 12, 24, 36, and 48 months, respectively. During the follow-up period, 33 patients died: 23 due to HCC, 7 due to hepatic failure, 2 from other diseases, and 1 of unknown causes.

Table 3 shows the results of univariate and multivariate analyses of prognostic factors for survival. The analyses showed that elevated serum endocan, AFP, and DCP levels were significantly associated with poor survival; however, VEGF/platelets did not reach statistical significance. Multivariate analysis showed that elevated serum endocan $(\geq 2.20 \mathrm{ng} / \mathrm{mL})$ levels, poor hepatic function, advanced tumor stage, and non-curative treatment were independently prognostic of poor survival. The cumulative survival rates were significantly lower in patients with higher versus lower serum endocan levels (Fig. 4A); similar results were observed when the patients were stratified by serum levels of AFP (Fig. 4B) and DCP (Fig. $4 \mathrm{C})$.

\section{Serum endocan level as a prognostic biomarker of tumor recurrence}

The present study also aimed to determine whether serum endocan, AFP, DCP, and VEGF/platelets could be prognostic factors for tumor recurrence. For this purpose, we performed a subgroup analysis using the curative treatment group (n $=38$ ). The analyses revealed that endocan, AFP, DCP, and VEGF/platelets were not statistically significant. Analyses using patients $(n=30)$ who received RFA also revealed that these serum markers had no significance. 

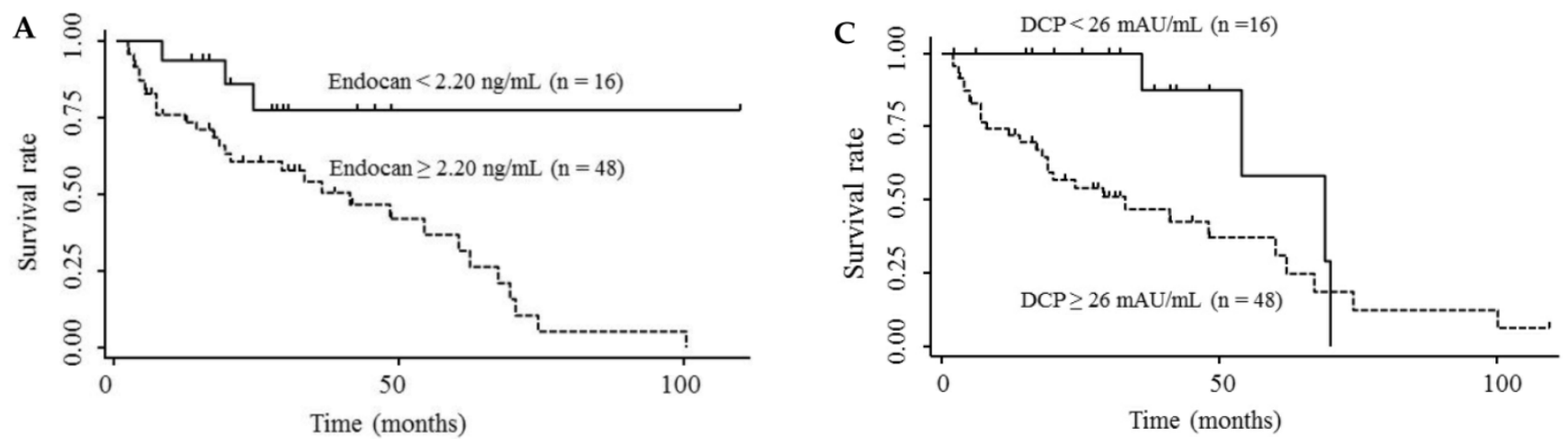

Figure 4. Kaplan-Meier survival curves in $\mathrm{HCC}$ patients with (A) serum endocan levels $\geq 2.20$ and $<2.20 \mathrm{ng} / \mathrm{mL}(P=0.019)$ and stratified by level of $(B)$

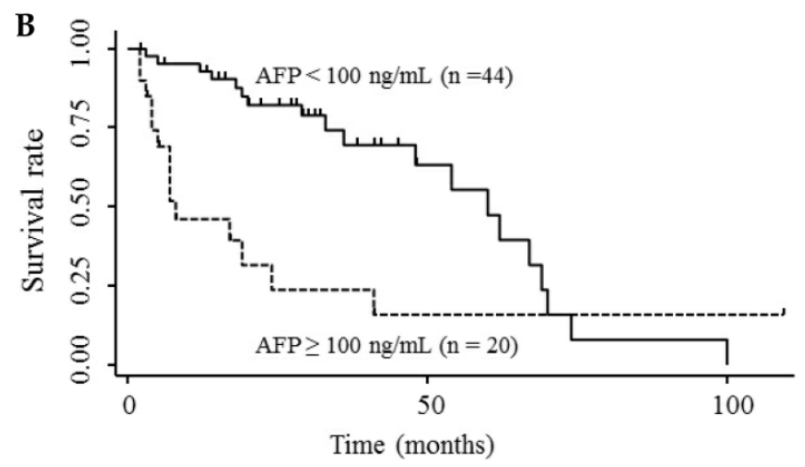
$\operatorname{AFP}(P=0.00 \mathrm{I})$ and $(C) \operatorname{DCP}(P=0.033)$. HCC, hepatocellular carcinoma; AFP, $\alpha$-fetoprotein; DCP, des- $\gamma$-carboxy prothrombin.

Table 3. Analysis of prognostic factors for survival in HCC patients $(n=64)$.

\begin{tabular}{|c|c|c|c|c|c|c|c|c|c|c|c|c|}
\hline \multirow[t]{2}{*}{ Variable } & \multirow[t]{2}{*}{ Category } & \multicolumn{2}{|c|}{ Univariate analysis } & \multicolumn{2}{|c|}{$\begin{array}{l}\text { Multivariate analysis } \\
\text { including serum endocan }\end{array}$} & \multicolumn{2}{|c|}{$\begin{array}{l}\text { Multivariate analysis } \\
\text { including serum AFP }\end{array}$} & \multicolumn{2}{|c|}{$\begin{array}{l}\text { Multivariate analysis } \\
\text { including serum DCP }\end{array}$} & \multicolumn{3}{|c|}{$\begin{array}{l}\text { Multivariate analysis } \\
\text { including serum endocan, } \\
\text { AFP, and DCP }\end{array}$} \\
\hline & & $\begin{array}{l}\text { Relative } \\
\text { hazard }\end{array}$ & $P$-value* & $\begin{array}{l}\text { Relative } \\
\text { hazard }\end{array}$ & $95 \% \mathrm{CI}$ & $\begin{array}{l}\text { Relative } \\
\text { hazard }\end{array}$ & $95 \% \mathrm{CI}$ & $\begin{array}{l}\text { Relative } \\
\text { hazard }\end{array}$ & $95 \% \mathrm{CI}$ & $\begin{array}{l}\text { Relative } \\
\text { hazard }\end{array}$ & $95 \%$ CI & $\begin{array}{l}P \text {-value } \\
*\end{array}$ \\
\hline \multirow[t]{2}{*}{ Age (years) } & $<70$ & 1 & & 1 & & 1 & & 1 & & 1 & & \\
\hline & $\geq 70$ & 1.21 & $0.84-1.790 .308$ & 1.43 & $0.94-2.250 .097$ & 1.60 & $1.04-2.540 .034$ & 1.24 & $0.79-2.000 .355$ & 1.39 & $0.89-2.22$ & 0.154 \\
\hline \multirow[t]{2}{*}{ Gender } & Female & 1 & & 1 & & 1 & & 1 & & 1 & & \\
\hline & Male & 0.96 & $0.67-1.420 .839$ & 0.97 & $0.61-1.540 .888$ & 0.97 & $0.61-1.540 .886$ & 0.84 & $0.51-1.370 .480$ & 0.91 & $0.57-1.47$ & 0.705 \\
\hline \multirow{2}{*}{$\begin{array}{l}\text { Etiology of liver } \\
\text { disease }\end{array}$} & Non-viral & 1 & & 1 & & 1 & & 1 & & 1 & & \\
\hline & Viral & 0.79 & $0.54-1.240 .293$ & 0.84 & $0.51-1.400 .495$ & 0.64 & $0.40-1.050 .078$ & 0.67 & $0.42-1.110 .117$ & 0.80 & $0.48-1.36$ & 0.396 \\
\hline \multirow[t]{2}{*}{ Child-Pugh grade } & A & 1 & & 1 & & 1 & & 1 & & 1 & & \\
\hline & $\mathrm{B} / \mathrm{C}$ & 1.34 & $0.94-1.910 .105$ & 1.59 & $0.99-2.610 .056$ & 1.92 & $1.18-3.150 .009$ & 1.66 & $1.04-2.720 .032$ & 1.66 & $1.05-2.67$ & 0.032 \\
\hline \multirow[t]{2}{*}{ Tumor stage } & $\mathrm{I} / \mathrm{II}$ & 1 & & 1 & & 1 & & 1 & & 1 & & \\
\hline & III/IV & 1.61 & $1.12-2.290 .012$ & 2.72 & $1.60-4.760 .0002$ & 1.48 & $0.85-2.600 .168$ & 1.89 & $1.17-3.080 .009$ & 1.94 & $1.03-3.78$ & 0.040 \\
\hline \multirow[t]{2}{*}{ Treatment option } & Curative & 1 & & 1 & & 1 & & 1 & & 1 & & \\
\hline & Non-curative & 2.08 & $1.45-3.070 .0001$ & 1.75 & $1.19-2.630 .004$ & 1.81 & $1.24-2.710 .002$ & 2.14 & $1.42-3.340 .0002$ & 1.66 & $1.10-2.60$ & 0.014 \\
\hline \multirow{2}{*}{$\begin{array}{l}\text { Serum endocan } \\
(\mathrm{ng} / \mathrm{mL})\end{array}$} & $<2.20$ & 1 & & 1 & & & & & & 1 & & \\
\hline & $\geq 2.20$ & 1.95 & $1.16-3.980 .009$ & 2.36 & $1.22-5.360 .008$ & & & & & 2.46 & $1.24-5.78$ & 0.008 \\
\hline \multirow{2}{*}{$\begin{array}{l}\text { VEGF/platelet } \\
\left(\mathrm{pg} / 10^{6}\right)\end{array}$} & $<2.18$ & 1 & & & & & & & & & & \\
\hline & $\geq 2.18$ & 1.02 & $0.99-1.040 .127$ & & & & & & & & & \\
\hline \multirow{2}{*}{$\begin{array}{l}\text { Serum AFP } \\
(\mathrm{ng} / \mathrm{mL})\end{array}$} & $<100$ & 1 & & & & 1 & & & & 1 & & \\
\hline & $\geq 100$ & 1.76 & $1.22-2.500 .003$ & & & 1.76 & $1.00-3.110 .049$ & & & 1.60 & $0.86-2.96$ & 0.142 \\
\hline \multirow{2}{*}{$\begin{array}{l}\text { Serum DCP } \\
(\mathrm{mAU} / \mathrm{mL})\end{array}$} & $<26$ & 1 & & & & & & 1 & & 1 & & \\
\hline & $\geq 26$ & 1.73 & $1.08-3.18 \quad 0.021$ & & & & & 1.90 & $1.07-3.790 .027$ & 1.64 & $0.94-3.22$ & 0.081 \\
\hline
\end{tabular}

HCC, hepatocellular carcinoma; VEGF, vascular endothelial growth factor; AFP, $\alpha$-fetoprotein; DCP, des- $\gamma$-carboxy prothrombin; CI, confidence interval.

* Cox proportional hazard model. 


\section{Prognostic stratification of serum endocan combined with tumor markers}

Based on the results showing that serum endocan, AFP, and DCP were independent prognostic biomarkers for survival, we tested whether the combination of endocan with these other markers can result in better prognostic stratification. Prognostic stratification was improved when serum endocan was combined with AFP or DCP (compared with any individual marker), and increased numbers of elevated
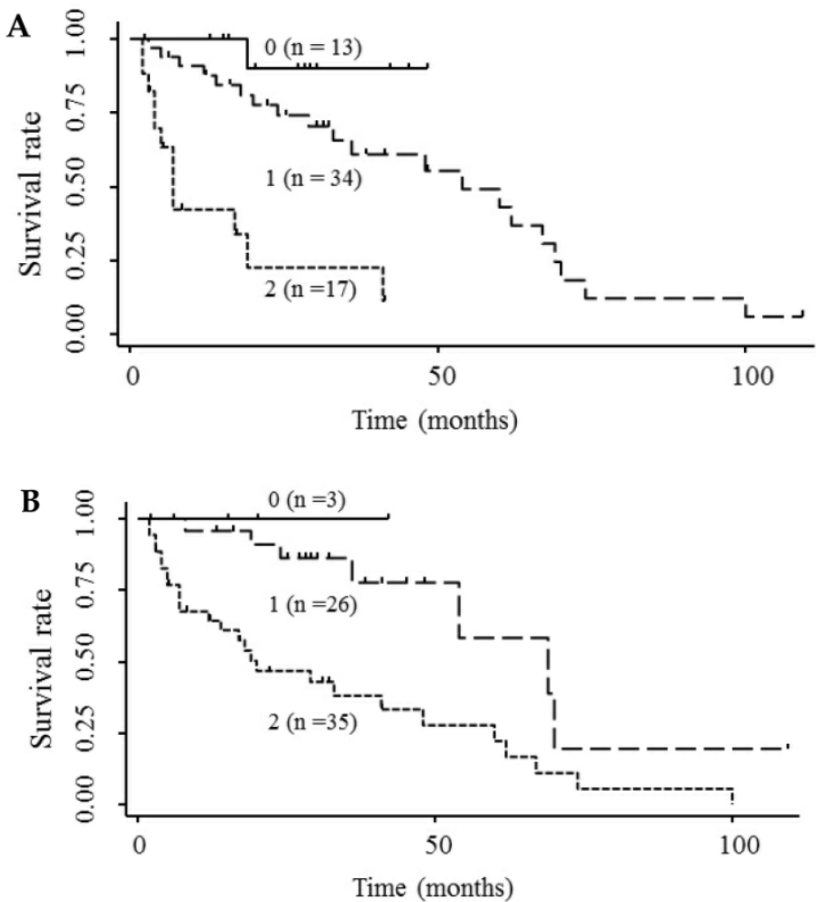

serum markers predicted worse survival (Fig. 5A and 5B). Similarly, the combination of serum AFP and DCP was more prognostic than either alone (Fig. 5C), and the elevation of all three markers was more predictive of worse survival than any individual marker or pair of markers (Fig. 5D). Based on multivariate analysis with the Cox proportional hazard model, two or three elevated serum markers were significantly associated with poor survival (Table 4 ).
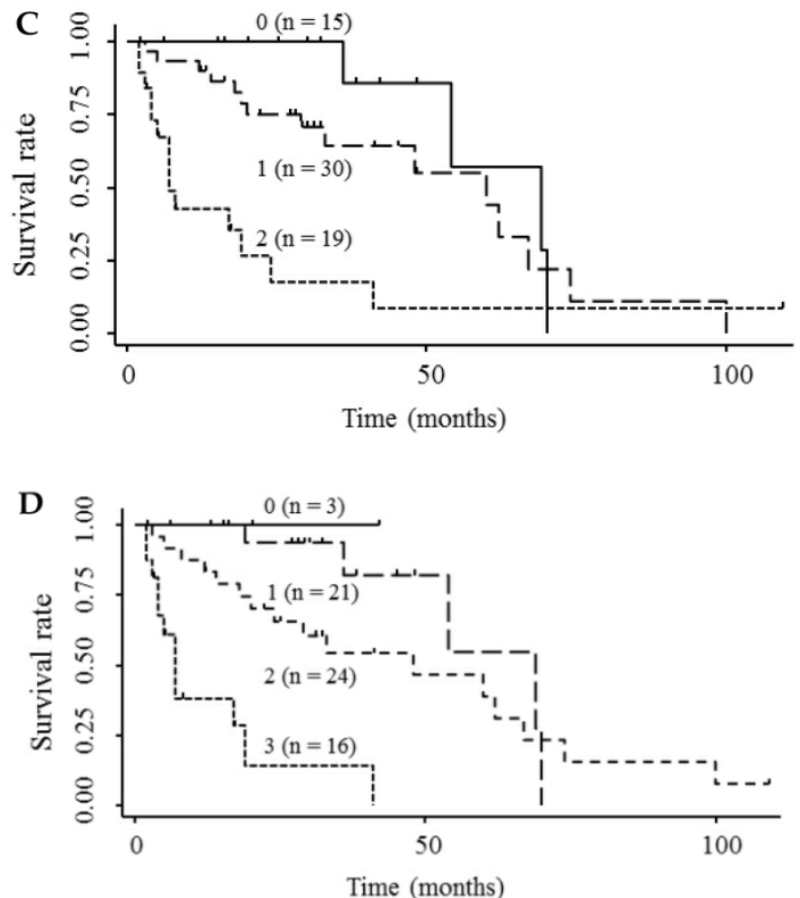

Figure 5. Kaplan-Meier survival curves among $\mathrm{HCC}$ patient groups according to the number of elevated serum markers. $(\mathrm{A})$ Endocan and $\mathrm{AFP}(\mathrm{P}<0.000 \mathrm{I})$. (B) Endocan and DCP $(P=0.002)$. (C) AFP and DCP $(P=0.0008)$. (D) Endocan, AFP, and DCP $(P<0.000 \mathrm{I})$. HCC, hepatocellular carcinoma; AFP, $\alpha$-fetoprotein; DCP, des- $\gamma$-carboxy prothrombin.

Table 4. Relative hazard according to the number of elevated serum markers $(n=64)$.

\begin{tabular}{|c|c|c|c|c|c|c|c|c|}
\hline \multirow[t]{2}{*}{ Variable } & \multirow{2}{*}{$\begin{array}{l}\text { Number of } \\
\text { elevated } \\
\text { markers }\end{array}$} & \multirow{2}{*}{$\begin{array}{l}\text { No. of } \\
\text { patients }\end{array}$} & \multicolumn{3}{|c|}{ Univariate analysis } & \multicolumn{3}{|c|}{ Multivariate analysis } \\
\hline & & & Relative hazard & $95 \% \mathrm{CI}$ & $P$-value* & Relative hazard & $95 \% \mathrm{CI}$ & $P$-value* \\
\hline \multirow[t]{3}{*}{ Serum endocan and AFP } & 0 & 13 & 1 & & & 1 & & \\
\hline & 1 & 34 & 4.22 & $0.55-32.62$ & 0.168 & 2.66 & $0.32-21.99$ & 0.364 \\
\hline & 2 & 17 & 21.90 & $2.82-170.29$ & 0.003 & 12.07 & $1.41-103.66$ & 0.023 \\
\hline \multirow[t]{2}{*}{ Serum endocan and DCP } & 0 or 1 & 29 & 1 & & & 1 & & \\
\hline & 2 & 35 & 4.06 & $1.75-9.39$ & 0.001 & 4.26 & $1.63-11.14$ & 0.003 \\
\hline \multirow[t]{3}{*}{ Serum endocan, AFP, and DCP } & 0 or 1 & 24 & 1 & & & 1 & & \\
\hline & 2 & 24 & 2.19 & $0.78-6.17$ & 0.137 & 1.90 & $0.64-5.62$ & 0.246 \\
\hline & 3 & 16 & 14.63 & $4.82-44.43$ & $<0.0001$ & 10.31 & $2.64-40.23$ & 0.001 \\
\hline
\end{tabular}

CI, confidence interval; AFP, $\alpha$-fetoprotein; DCP, des- $\gamma$-carboxy prothrombin.

* Cox proportional hazard model. Variables assessed in the multivariate analysis included age (< 70 years vs. $\geq 70$ years), gender (female vs. male), etiology of liver disease (non-viral vs. viral), tumor stage (stage I/II vs. III/IV), Child-Pugh grade (A vs. B/C), treatment option, and number of elevated markers. 


\section{Discussion}

Serum endocan levels have been reported to vary in healthy subjects, and our results (median, $1.145 \mathrm{ng} / \mathrm{mL}$ ) were similar or somewhat higher than those reported in previous studies (mean, 0.43-1.08 $\mathrm{ng} / \mathrm{mL}$; median, $0.3-0.77 \mathrm{ng} / \mathrm{mL})(6,14,18,19)$. Our finding that serum endocan levels were higher in HCC versus non-HCC patients is in agreement with the results of recent studies $(23,25)$. Elevated serum endocan levels have also been observed in patients with non-small cell lung cancer, clear-cell renal cell carcinoma, colorectal cancer, and invasive bladder cancer $(14,16,18,19)$.

The present study suggests a close relationship between elevated serum endocan levels and poor survival in HCC patients. The same relationship has been reported in studies of patients with non-small cell lung cancer and colorectal cancer $(14,16)$. In our cohort, approximately $90 \%$ of the HCC patients died due to liver-related causes, including HCC and hepatic failure. Hence, our results indicate that serum endocan levels can predict liver-related mortality in HCC patients.

In contrast to our results, a recent study has shown that elevated serum endocan levels were not significantly related to poor survival in alcoholic LC patients with HCC, although univariate analysis revealed that the endocan levels in patients with advanced HCC reached statistical significance (25). These somewhat variable results may have occurred due to the different patient characteristics, including the different etiologies of liver disease. Another explanation may be the different cut-off values used for serum endocan measurements. The serum endocan cut-off value of $5 \mathrm{ng} / \mathrm{mL}$ in the study of alcoholic patients was considerably higher than the corresponding value of $2.2 \mathrm{ng} / \mathrm{mL}$ that was used in our study. The present study suggests that the relatively low serum endocan cut-off value can distinguish HCC survivors from nonsurvivors. Future studies with other cohorts must be performed to verify our results. Additionally, in the study of alcoholic patients, it is possible that serum endocan was not statistically significant because it was analyzed together with serum syndecan-1, which is more strongly prognostic of survival in advanced HCC patients.

We found that elevated serum endocan levels were correlated with a higher number of tumors and with vascular invasion, indicating a close link between serum endocan levels and HCC aggressiveness. A previous study showed that endocan mRNA and protein were overexpressed in HCC tumor vessels and that endocan mRNA levels were correlated with intra-tumoral microvessel density and vascular inva- sion (22). An immunohistological study of HCC also found a close relationship between endocan expression and microvascular invasion (24). Moreover, microvessel density in surgically resected HCCs was found to be predictive of poor patient survival (21), as patients with hypervascular HCC had worse survival outcomes than those with hypovascular HCC (31). Taken together, these findings suggest that serum endocan levels reflect HCC aggressiveness based on the endothelial proliferation of tumor vessels, thus influencing patient survival.

We also found that elevated serum endocan levels were closely associated with poor hepatic function in both HCC and LC patients. Simultaneously, serum endocan levels were higher in HCC versus LC patients at each grade of hepatic function. Similar results were shown in a recent study of alcoholic LC patients (25). Angiopoietin-2, another vascular endothelium-derived factor $(32,33)$, was also shown to be expressed at higher levels in HCC patients compared with LC patients (34). However, in contrast to endocan, serum angiopoietin levels were similar in patients with different grades of hepatic function. Because endocan levels may reflect hepatic function as well as HCC aggressiveness, this factor may be specifically helpful in predicting survival in HCC patients, as most of these patients have LC. The mechanisms responsible for elevated serum endocan levels in non-HCC patients with LC and the positive relationship between serum endocan levels and grades of hepatic function remain unclear. These mechanisms may be elucidated by assaying the expression of endocan during angiogenesis related to LC progression and by analyzing the metabolism of this proteoglycan.

Recent studies have shown that endocan is closely related to VEGF-A, which is a central regulator of angiogenesis (7-9). In a study of cultured vascular endothelial cells and VEGF-A transgenic mice, it was found that VEGF-A stimulates endocan secretion by endothelial cells, which in turn enhances VEGF-A-induced endothelial migration and angiogenesis (19). Furthermore, a recent study of bladder cancer revealed a significant correlation between serum VEGF and endocan levels (19). Given these findings, we expected that there would be a similar correlation in our patients; however, we did not observe such a correlation. A possible reason for this result is that in the case of HCC, serum endocan levels can be influenced by not only serum VEGF levels but also by the severity of hepatic function impairment. Although many studies have demonstrated the predictive value of serum VEGF for survival in HCC patients (3), the present study suggested that serum endocan, rather than serum VEGF, can predict patient survival. To verify these findings, further analyses 
with a larger number of HCC patients are required.

Our findings indicate that serum endocan levels may be complementary to serum AFP and DCP levels for predicting survival in HCC patients. The latter two proteins were shown to be prognostic biomarkers for survival in patients at different stages of HCC who were receiving various types of treatment $(35,36)$. Indeed, serum AFP and DCP, alone or in combination, could allow prognostic stratification in our cohort. Although serum endocan and AFP levels were weakly correlated, serum endocan, but not AFP (data not shown), was specifically associated with grade of hepatic function. Our results suggest that serum endocan may be a new prognostic biomarker in patients with HCC and may contribute to better prognostic stratification when combined with AFP and/or DCP. Serum endocan, as well as AFP and DCP, should be routinely measured for the appropriate management of HCC, although further studies are needed to confirm the utility of these combinations of markers.

In our cohort, serum endocan, AFP, DCP, and VEGF/platelets were not effective prognostic factors for tumor recurrence in HCC patients who underwent curative treatments. However, previous studies have demonstrated the predictive value of elevated serum AFP, DCP, and VEGF levels for HCC recurrence (37-41). Because the relatively small number of examined patients might weaken the statistical power, larger-scale studies are warranted to determine the relationship between tumor recurrence and serum endocan as well as other serum markers.

Experimental and clinical studies demonstrated that some types of cancer cells as well as tumor endothelial cells secrete endocan (10-13). Inhibition of endocan gene expression via small interfering RNA was found to inhibit the nuclear factor- $\mathrm{kB}$ pathway and to induce cell cycle arrest by induction of the phosphatase and tensin homolog, leading to inhibition of migration and invasion of HCC or colorectal cancer cells $(23,42)$. These findings suggest that endocan may be a therapeutic target in cancer patients, as tumor progression may be inhibited by suppressing endocan secretion or function.

Our results suggest that serum endocan may serve as a prognostic biomarker for survival in HCC patients. Furthermore, the combination of serum endocan levels with serum AFP and/or DCP levels can result in better prognostic stratification of these patients, which may be helpful in planning therapeutic management strategies. Although our findings should be verified in a larger cohort, they encourage further investigation of the utility of serum endocan as a predictive biomarker for each treatment option and a pharmacodynamic biomarker in anticancer drug therapies.

\section{Acknowledgements}

We thank Mr. Mitsuru Araya for his excellent technical support. This study was fully funded by the Department of Hepatology, Kanazawa Medical University, Ishikawa, Japan.

\section{Conflict of interest}

We declare that there are no conflicts of interest associated with this work.

\section{References}

1. Patel M, Shariff M I, Ladep N G, et al. Hepatocellular carcinoma: diagnostics and screening. J Eval Clin Pract. 2012; 18: 335-42.

2. Padhya K T, Marrero J A, Singal A G. Recent advances in the treatment of hepatocellular carcinoma. Curr Opin Gastroenterol. 2013; 29: 285-92.

3. Schoenleber S J, Kurtz D M, Talwalkar J A, Roberts L R, Gores G J. Prognostic role of vascular endothelial growth factor in hepatocellular carcinoma: systematic review and meta-analysis. Br J Cancer. 2009; 100: 1385-92.

4. Miura S, Mitsuhashi N, Shimizu H, et al. Fibroblast growth factor 19 expression correlates with tumor progression and poorer prognosis of hepatocellular carcinoma. BMC Cancer. 2012; 12: 56.

5. Lassalle P, Molet S, Janin A, et al. ESM-1 is a novel human endothelial cell-specific molecule expressed in lung and regulated by cytokines. J Biol Chem. 1996; 271: 20458-64.

6. Bechard D, Meignin V, Scherpereel A, et al. Characterization of the secreted form of endothelial-cell-specific molecule 1 by specific monoclonal antibodies. J Vasc Res. 2000; 37: 417-25.

7. Sarrazin S, Adam E, Lyon M, et al. Endocan or endothelial cell specific molecule-1 (ESM-1): a potential novel endothelial cell marker and a new target for cancer therapy. Biochim Biophys Acta. 2006; 1765: 25-37.

8. Abid M R, Yi X, Yano K, Shih S C, Aird W C. Vascular endocan is preferentially expressed in tumor endothelium. Microvasc Res. 2006; 72: 136-45.

9. Rennel E, Mellberg S, Dimberg A, et al. Endocan is a VEGF-A and PI3K regulated gene with increased expression in human renal cancer. Exp Cell Res. 2007; 313: 1285-94.

10. Scherpereel A, Gentina T, Grigoriu B, et al. Overexpression of endocan induces tumor formation. Cancer Res. 2003; 63: 6084-9.

11. Almog N, Ma L, Raychowdhury R, et al. Transcriptional switch of dormant tumors to fast-growing angiogenic phenotype. Cancer Res. 2009; 69: 836-44.

12. Maurage C A, Adam E, Mineo J F, et al. Endocan expression and localization in human glioblastomas. J Neuropathol Exp Neurol. 2009; 68: 633-41.

13. Cornelius A, Cortet-Rudelli C, Assaker R, et al. Endothelial expression of endocan is strongly associated with tumor progression in pituitary adenoma. Brain Pathol. 2012; 22: 757-64.

14. Grigoriu B D, Depontieu F, Scherpereel A, et al. Endocan expression and relationship with survival in human non-small cell lung cancer. Clin Cancer Res. 2006; 12: 4575-82.

15. Liu N, Zhang L H, Du H, et al. Overexpression of endothelial cell specific molecule-1 (ESM-1) in gastric cancer. Ann Surg Oncol. 2010; 17: 2628-39.

16. Ji N Y, Kim Y H, Jang Y J, et al. Identification of endothelial cell-specific molecule-1 as a potential serum marker for colorectal cancer. Cancer Sci. 2010; 101: 2248-53.

17. Kim J H, Park M Y, Kim C N, et al. Expression of endothelial cell-specific molecule-1 regulated by hypoxia inducible factor-1alpha in human colon carcinoma: impact of ESM-1 on prognosis and its correlation with clinicopathological features. Oncol Rep. 2012; 28: 1701-8.

18. Leroy X, Aubert S, Zini L, et al. Vascular endocan (ESM-1) is markedly overexpressed in clear cell renal cell carcinoma. Histopathology. 2010; 56: 180-7.

19. Roudnicky F, Poyet C, Wild P, et al. Endocan is upregulated on tumor vessels in invasive bladder cancer where it mediates VEGF-A-induced angiogenesis. Cancer Res. 2013; 73: 1097-106.

20. El Behery M M, Seksaka M A, Ibrahiem M A, Saleh H S, El Alfy Y. Clinicopathological correlation of endocan expression and survival in epithelial ovarian cancer. Arch Gynecol Obstet. 2013.

21. Huang G W, Tao Y M, Ding X. Endocan expression correlated with poor survival in human hepatocellular carcinoma. Dig Dis Sci. 2009; 54: 389-94.

22. Chen L Y, Liu X, Wang S L, Qin C Y. Over-expression of the Endocan gene in endothelial cells from hepatocellular carcinoma is associated with angiogenesis and tumour invasion. J Int Med Res. 2010; 38: 498-510.

23. Kang Y H, Ji N Y, Lee C I, et al. ESM-1 silencing decreased cell survival, migration, and invasion and modulated cell cycle progression in hepatocellular carcinoma. Amino Acids. 2011; 40: 1003-13.

24. Ziol M, Sutton A, Calderaro J, et al. ESM-1 Expression in Stromal Cells is Predictive for Recurrence after Radiofrequency Ablation in Early Hepatocellular Carcinoma. J Hepatol. 2013.

25. Nault J C, Guyot E, Laguillier C, et al. Serum Proteoglycans as Prognostic Biomarkers of Hepatocellular Carcinoma in Patients with Alcoholic Cirrhosis. Cancer Epidemiol Biomarkers Prev. 2013; 22: 1343-52. 
26. Minagawa M, Ikai I, Matsuyama Y, Yamaoka Y, Makuuchi M. Staging of hepatocellular carcinoma: assessment of the Japanese TNM and AJCC/UICC TNM systems in a cohort of 13,772 patients in Japan. Ann Surg. 2007; 245: 909-22.

27. Kokudo N, Makuuchi M. Evidence-based clinical practice guidelines for hepatocellular carcinoma in Japan: the J-HCC guidelines. J Gastroenterol. 2009; 44 Suppl 19: 119-21.

28. Gunsilius E, Petzer A, Stockhammer G, et al. Thrombocytes are the major source for soluble vascular endothelial growth factor in peripheral blood. Oncology. 2000; 58: 169-74.

29. Shim J H, Park J W, Kim J H, et al. Association between increment of serum VEGF level and prognosis after transcatheter arterial chemoembolization in hepatocellular carcinoma patients. Cancer Sci. 2008; 99: 2037-44.

30. Tyson G L, Duan Z, Kramer J R, et al. Level of alpha-fetoprotein predicts mortality among patients with hepatitis C-related hepatocellular carcinoma. Clin Gastroenterol Hepatol. 2011; 9: 989-94.

31. Toyoda H, Kumuda T, Nakano S, et al. Significance of tumor vascularity as a predictor of long-term prognosis in patients with small hepatocellular carcinoma treated by percutaneous ethanol injection therapy. J Hepatol. 1997; 26: 1055-62.

32. Maisonpierre P C, Suri C, Jones P F, et al. Angiopoietin-2, a natural antagonist for Tie2 that disrupts in vivo angiogenesis. Science. 1997; 277: 55-60.

33. Augustin H G, Koh G Y, Thurston G, Alitalo K. Control of vascular morphogenesis and homeostasis through the angiopoietin-Tie system. Nat Rev Mol Cell Biol. 2009; 10: 165-77.

34. Scholz A, Rehm V A, Rieke S, et al. Angiopoietin-2 serum levels are elevated in patients with liver cirrhosis and hepatocellular carcinoma. Am J Gastroenterol. 2007; 102: 2471-81.

35. Shimada M, Takenaka K, Fujiwara Y, et al. Des-gamma-carboxy prothrombin and alpha-fetoprotein positive status as a new prognostic indicator after hepatic resection for hepatocellular carcinoma. Cancer. 1996; 78: 2094-100.

36. Toyoda H, Kumada T, Kiriyama S, et al. Prognostic significance of simultaneous measurement of three tumor markers in patients with hepatocellular carcinoma. Clin Gastroenterol Hepatol. 2006; 4: 111-7.

37. Shiina S, Tateishi R, Arano T, et al. Radiofrequency ablation for hepatocellular carcinoma: 10-year outcome and prognostic factors. Am J Gastroenterol. 2012; 107: 569-77; quiz 78

38. Toyoda H, Kumada T, Tada T, et al. Prognostic significance of a combination of pre- and post-treatment tumor markers for hepatocellular carcinoma curatively treated with hepatectomy. J Hepatol. 2012; 57: 1251-7.

39. Poon R T, Lau C, Pang R, et al. High serum vascular endothelial growth factor levels predict poor prognosis after radiofrequency ablation of hepatocellular carcinoma: importance of tumor biomarker in ablative therapies. Ann Surg Oncol. 2007; 14: 1835-45.

40. Tamesa T, lizuka N, Mori N, et al. High serum levels of vascular endothelial growth factor after hepatectomy are associated with poor prognosis in hepatocellular carcinoma. Hepatogastroenterology. 2009; 56: 1122-6.

41. Zhong C, Wei W, Su X K, et al. Serum and tissue vascular endothelial growth factor predicts prognosis in hepatocellular carcinoma patients after partial liver resection. Hepatogastroenterology. 2012; 59: 93-7.

42. Kang Y H, Ji N Y, Han S R, et al. ESM-1 regulates cell growth and metastatic process through activation of NF-kappaB in colorectal cancer. Cell Signal. 2012; 24: 1940-9. 